\title{
Tool Development of Construction Project Auxiliary Green Land Area Audit Based on AutoCAD .NET API
}

\author{
Liu Yanmin ${ }^{1,2}$, Zheng Hui ${ }^{2,}$, Ma Xiaokang ${ }^{2}$ \\ ${ }^{1}$ Faculty of Engineering, China University of Geosciences, Wuhan, China \\ ${ }^{2}$ Information Center, Wuhan Geotechnical Engineering and Surveying Co. Ltd., Wuhan, China
}

\section{Email address:}

liuym85@163.com (Liu Yanmin), zhenghui2915@163.com (Zheng Hui),mxk32718409@163.com (Ma Xiaokang)

*Corresponding author

\section{To cite this article:}

Liu Yanmin, Zheng Hui, Ma Xiaokang. Tool Development of Construction Project Auxiliary Green Land Area Audit Based on AutoCAD .NET API. Science Discovery. Vol. 5, No. 7, 2017, pp. 587-591. doi: 10.11648/j.sd.20170507.29

Received: September 30, 2017; Accepted: November 14, 2017; Published: December 29, 2017

\begin{abstract}
The scheme of construction project is joint approved by planning department and landscape department in Wuhan. The planning green land ratio of the construction project must achieve the standard of auxiliary green land ratio. During the auxiliary green land audit, the area of all the green land entity in the planning map is classified according to different conversion factors. This paper has developed an adult tool for Construction project auxiliary green land area based on AutoCAD .NET API. Take advantage of this tool the classification and statistics of all kinds of green land entity can quickly and accurately complete, greatly reducing the workload of manual statistics and improving the accuracy of statistics, the efficiency of supporting green area audit can also greatly improved.
\end{abstract}

Keywords: AutoCAD, C\#, .NET API, Green land Ratio, Area Audit, Custom Development

\section{基于AutoCAD .NET API的建设工程配套绿地面积审核工具开发}

刘艳敏 $^{1,2}$, 郑晖 ${ }^{2 *}$, 马小康 ${ }^{2}$

${ }^{1}$ 工程学院, 中国地质大学 (武汉), 武汉, 中国

2信息中心，武汉市勘察设计有限公司，武汉，中国

邮箱

liuym85@163.com (刘艳敏), zhenghui2915@163.com（郑晖）, mxk32718409@163.com（马小康）

摘要: 武汉市建设工程项目的规划方案需经规划部门和园林部门并联审批。项目的规划绿地面积需达到建设工程项目 配套绿地率标准。配套绿地面积审核时需对规划方案总平面图中的所有绿地实体按不同的折算系数分类汇总后计算规 划绿地率。本文基于AutoCAD .NET API开发了建设工程配套绿地面积审核工具, 使用该工具可快速准确实现各类绿地 实体面积的分类汇总统计, 完成绿地率指标与规划绿地率的比对, 可大大减少了人工统计的工作量并提高了统计的准 确性，能够大幅提高配套绿地面积审核的效率。

关键词：AutoCAD，C\#，.NET API，绿地率，面积审核，定制开发 


\section{1. 引言}

城市绿地系统具有巨大的生态效益、景观效益和经济 效益。如果城市景观优美, 居住环境雅致, 会提高城市的 知名度和居民的幸福感, 吸引更多的客商来投资兴业, 进 而促进当地经济的蓬勃发展。经济的发展又为城市绿化建 设提供坚实的经济基础, 促使城市绿化迈向更高层次, 也 就促进了城市的生态建设的良性循环和稳步发展。城市绿 化建设中不仅关系绿地美化、观赏、休㮩等功能, 更加注 重绿地生态系统服务、保护景观多样性等综合功能, 城市 绿地已成为衡量城市地区生态可持续的重要标志 [1-2]。现 在通常采用城市绿地率 (公园绿地、生产绿地、防护绿地、 附属绿地、其他绿地) 这一重要指标来衡量一个城市城市 绿地水平。城市绿地率对加强绿化生态建设, 扩大绿化面 积, 提高绿地覆盖率, 优化绿地结构具有重要意义。

当前, 武汉市建设工程项目配套绿地面积审核时, 由 建设单位提交该项目的规划（建筑）设计方案总平面图以 及总平面图基础上的绿化布置图。在这两个图中不仅要确 定各绿地实体的位置、形状和大小，还应明确绿地的表现 形式（露天绿化、垂直绿化、屋顶绿化等）。实际建设工 程配套绿地面积审核时, 需在总平面图上对各类绿地实体 进行人工分类, 然后再按照折算系数进行绿地面积的汇总 统计, 最后将计算规划绿地率与绿地率指标进行比较, 判 断建设项目规划方案是否达标[3]。该方法人工依赖性强, 不仅繁复效率低下, 而且易出错难纠错。由于绿地面积审 核是在AutoCAD环境下进行的, 本文基于AutoCAD .NET API进行定制开发, 形成一套绿地面积分类汇总工具来辅助 审核人员快速作业，使用该工具既可以提高审核工作效率 还可以减少审核出现的错误, 因而具有较强的实用价值。

\section{AutoCAD .NET API概述}

\subsection{AutoCAD二次开发方法比较}

目前 AutoCAD二次开发的工具比较多, 如 Visual Lisp、VBA和ObjectARX等。它们各有优缺点: Visual Lisp
采用Lisp语言开发，简单但不直观; VBA采用Visual Basic 语言开发, 开发简单方便但功能不足; 而ObjectARX是 基于Visual C++开发语言的, 功能强但难以掌握 [4-6]。

从AutoCAD 2006开始, AutoCAD就增加了.NET API, 它的出现使用户可通过编程的方式利用程序集和类库操 作AutoCAD应用程序和图形文件。使用许多不同的编程语 言和环境都可访问AutoCAD .NET API中开放的对象。采 用.NET API进行AutoCAD具有以下优点[7]:

1) 有更多的编程环境可以访问AutoCAD图形。在此之 前, 开发人员只能使用ActiveX Automation和支持 COM、AutoLISP以及包含 ObjectARX的 $\mathrm{C}++$ 的语 言。

2) 通过使用应用程序的原生.NET API或公开的 ActiveX/COM库, 与其他基于Windows的应用程序 （如Microsoft Excel和Word）集成更加容易。

3) .NET框架可兼容32位及64位操作系统。

4) 与传统的C++编程语言相比.NET API可提供高级访 问接口, 并且学习成本也更低。

综上所述, 在.NET框架下利用.NET语言对AutoCAD 进行二次开发, 具有完全面向对象、方便易用的特点, 是 最合适的AutoCAD二次开发工具。

\section{2. AutoCAD .NET API对象模型}

AutoCAD .NET API 的重要组成部分是对象, AutoCAD程序或图形中的元素可使用DWG文件中的对象 来详细精确表示。DWG文件是一个包含有完整图形信息 的数据库, 其文件结构如图1所示 $[8]$ 。DWG文件中的各种 对象以程序集和命名空间进行分类。AutoCAD对象层次模 型如图2所示 [8], 可将其视为在AutoCAD环境下打开DWG 文件的状态。图1仅给出了DWG文件的结构, 图2相比图1 增加了AutoCAD环境下的菜单栏、工具栏、状态栏以及图 形编辑、管理等通用内容。

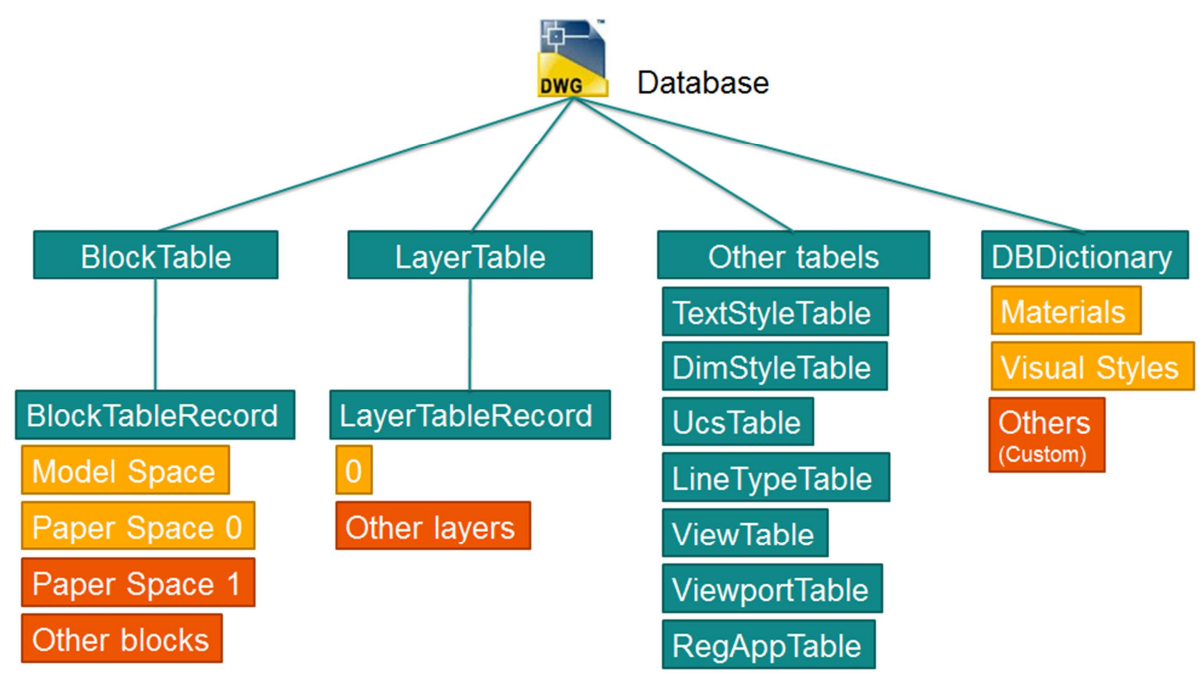

图1 DWG文件数据库结构。 


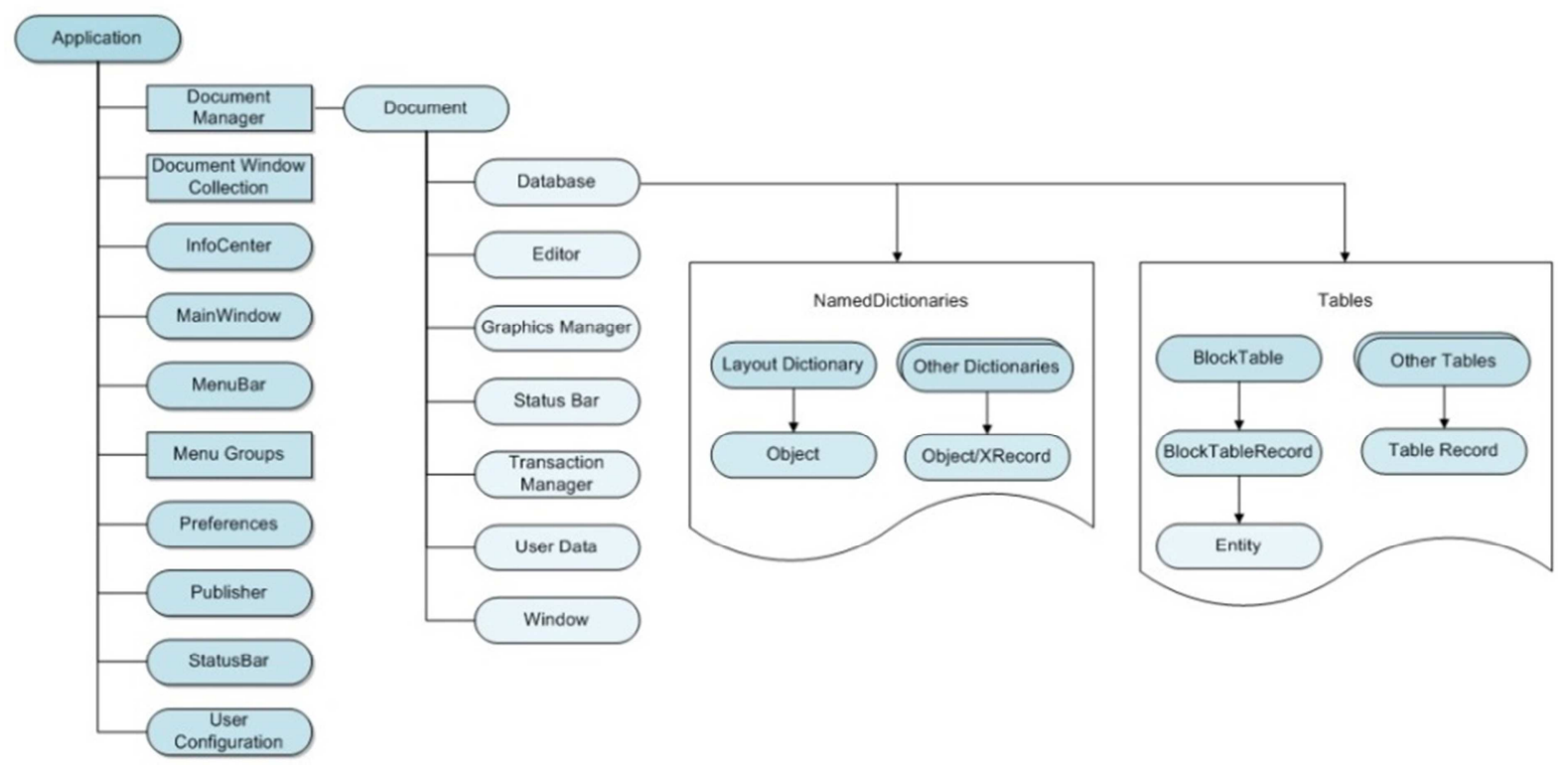

图2 AutoCAD 对象层次结构图。

AutoCAD .NET API的它中包含图形对象和非图形对 象。图形对象就是我们所说的实体, 这些对象是组成图形 的点、线、面、标注等元素。通过引用块表记录可以在图 形中增加图形对象, 准确地获得的块表记录后使用对象的 方法和属性就可以修改和查询对象。每一个图形对象都具 有图形编辑常用的复制、删除、移动、镜像等方法。非图 形对象指的是那些图形中不可见的对象, 例如块、组、图 层、视图、线型、标注样式、表格样式等。另外, 为了扩 展AutoCAD图形的自定义属性信息, 可以使用扩展数据 XData为图形添加一定数量的自定义属性, 还可以使用字 典来扩充图形对象的非图形特征属性[9]。

\section{3. 定制开发插件的方法}

采用C\#.NET开发AutoCAD插件时, 首先在C\#集成 开发环境中创建一个类库工程, 其次根据功能需要向项 目中添加AutoCAD动态链接库 (*.dll) 的引用, 并设置 不将引用的类库复制到本地, 然后类文件中添加定制的 命令和功能实现代码, 随后编译代码生成定制开发项目 的类库文件, 最后打开AutoCAD应用程序后, 通过在命 令行中输入NETLOAD命令加载编译后的类库文件, 这样 在命令行中就能通过键入定制的命令名称来实现定制功 能。定制开发插件的开发过程如下图3所示[10]。

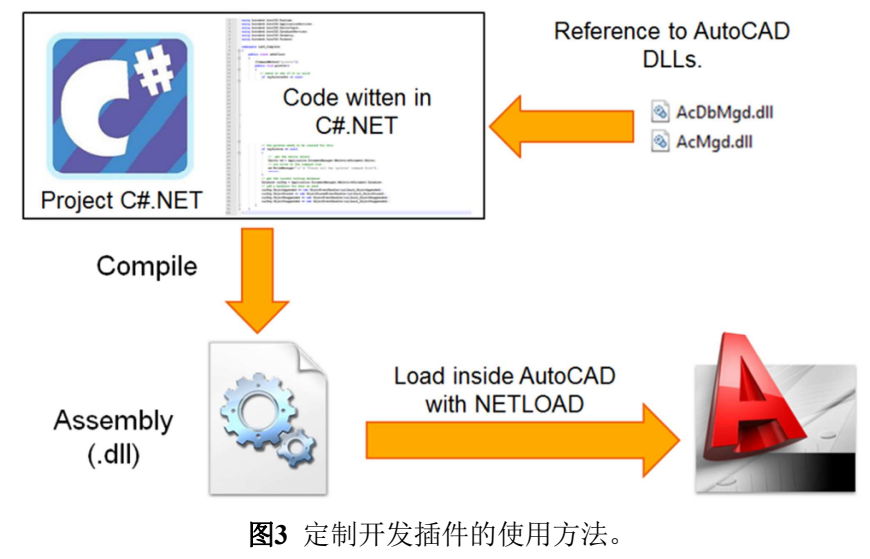

\section{3. 绿地面积审核工具定制开发}

\section{1. 业务模型}

武汉市建设工程项目规划方案由武汉市国土和规划 局及武汉市园林和林业局并联审核。建设单位需向先规 划部门提交项目规划设计方案, 规划部门对规划设计方 案中非绿地部分进行审核, 审核通过后再由园林部分对 规划设计方案中绿地部分进行审核。建设工程项目配套
绿地审核时, 需由建设单位提供其委托具有园林设计资 质的设计单位提供的总平面图和绿化布置图。园林部门 首先根据《武汉市城市绿化条例》并结合建设工程项目 的性质和用途确定该项目的配套绿地率; 其次在总平面 图上对绿地实体进行预处理并按照折算比例完成各类绿 地实体进行分类, 然后汇总所有折算后绿地的面积, 计 算该项目的规划绿地率, 参照该项目的配套绿地率指标 判断该项目的规划绿化率是否达标, 并将配套绿地审核 结果反馈给规划部门; 最后由规划部门综合并联审核意 
见出具该建设工程项目的审核意见。在建设工程项目竣 工验收规划条件核实阶段, 园林部门还应当对项目配套 绿化工程实施情况进行监督检查, 监督检查意见向规划 部门书面反馈。

\section{2. 功能规划}

根据上述业务模型, 绿地审核业务人员的工作重心 侧重于绿地实体图形的预处理和面积分类汇总。由于各 园林设计单位各有自己的绘图要求, 无法对总图中绿地 实体进行标准化, 导致总图上绿地实体的表达方式各不 一致。预处理的过程是检查绿地实体的绘制是否采用封 闭的多段线，并对不符合内容进行处理。面积的分类汇 总是对经预处理后符合标准的绿地实体进行面积汇总统 计。本文从实际审核场景出发, 对面积审核工具的功能 进行如下的规划:

1) 面积计算: 在每个绿地实体几何中心位置, 以绿 地实体所在图层的颜色, 采用指定的字体和字号在当前图 层上完成所有绿地实体的面积标注, 同时标注每一类折算 系数的绿地实体面积及所有绿地实体面积汇总之和。

2) 删除标注: 面积计算完成所有标注输出后, 如需 对绿地实体图形进行局部调整, 在重新计算面积之前, 需 对原标注进行删除, 否则新增的标注会覆盖在原标注上, 影响图形更改后的标注效果。

3）绿地填充: 按不同的折算系数对所有绿地实体采 用不同的样式进行填充, 起到美化图形的效果。

4)清除填充: 需要对部分绿地实体进行局部调整时, 应先清除已完成的填充, 确保调整后图形填充边界无误。

5) 列举实体: 将图形文件中所有的绿地实体列举出 来, 便于统计所有绿地实体的数量, 防止重复统计和遗漏 绿地实体。

6) 列举图层: 将图形文件中所有的图层列举出来, 便于检查是否按折算系数完成全部绿地实体的分类汇总 统计。

\section{3. 定制开发}

\section{3. 1. 新增标注实体}

首先获取DWG文档及对应的数据库对象并开启一个 事务, 然后创建要添加的实体对象, 按图1的结构获取实 体对象所在的大类以及该类下的实体记录对象, 然后完成 实体对象的各类属性的设置, 最后将创建实体添加到原实 体对象记录中, 再将该实体对象记录增加到事务中, 执行 该事务就可以完成实体对象的添加, 下面给出的是添加 MText类型标注实体实现过程。

//获取当前文档和数据库

Document acDoc $=$

Application.DocumentManager.MdiActiveDocument;

Database $\mathrm{acCurDb}=\mathrm{acDoc}$. Database;

using $($ Transaction acTrans $=$

acCurDb.TransactionManager.StartTransaction())

\{

BlockTable

acBlkTbl=acTrans.GetObject(acCurDb.BlockTableId,Open

Mode.ForRead) as BlockTable;
BlockTableRecord acBlkTblRec $=$

acTrans.GetObject(acBlkTbl[BlockTableRecord.ModelSpace ],OpenMode.ForWrite) as BlockTableRecord;

//创建标注对象

MText acMText = new MText();

acMText.Location $=$ textCenter;

acMText. Width $=40$;

acMText.Height $=5$;

acMText.Color $=$ layersColor[layIndex];

acMText.Contents

Math.Round(pPolyline.Area,2).ToString();

acBlkTblRec.AppendEntity(acMText);

acTrans.AddNewlyCreatedDBObject(acMText, true);

acTrans.Commit(); \}

\section{3.2. 绿地实体分类汇总统计}

绿地实体的预处理包含检查绿地实体是否以多段线 的形式闭合, 其次要按绿地实体的折算系数对所有绿地实 体进行分类。绿地实体的分类可按统一的颜色、图层、线 型、线宽等属性来判定，但由于颜色、线型、线宽具有较 多的选项值，不便于快速准确明确绿地实体分类。本文以 绿地面积折算系数作为图层名来直观区分各类绿地实体, 采用正则表达式来验证图层名确定绿地实体汇总统计分 析的范围。通过遍历DWG文件中的所有实体, 按实体编 号、折算系数、实体记录、颜色、图层等完成绿地实体面 积的汇总及标注。

Entity pEntity $=$ acTrans.GetObject(acObjTblRec, OpenMode.ForRead) as Entity;

if ((pEntity as Polyline) $!=$ null $)$

\{

Polyline pPolyline $=\mathrm{pEntity}$ as Polyline;

pPolyline.Close=true;

entityType $=p$ Polyline.Layer;

double calcType;

if (Regex.IsMatch(entityType,

@" $\left.\left.{ }^{\prime \wedge}[-] ?[0-9]+(\backslash \cdot[0-9]+) ? \$ "\right)\right)$

\{

calcType=Convert.ToDouble(entityType);

entityArea $=$ CalcLabEntityArea(entityIndex,calcType, acDoc, acBlkTblRec, pPolyline,entityLayers, layersColor);

\}

\subsection{3. 类库文件自动加载}

通过AutoCAD .NET API定制开发的功能被集成到 类库文件中。AutoCAD在打开是不会默认加载用户自定 义类库文件, 需要使用“NETLOAD”命令进行添加。为了 使AutoCAD启动后能够自动加载类库文件, 显示定制开 发的功能菜单, 需要找到AutoCAD安装目录X: $\backslash$ AutoCAD 2012 SSupport, 用记事本打开目录下的acad2012doc.lsp文 件, 并在该文件最后增加netload命令及类库文件路径, 保存后关闭该文件, 再次打开AutoCAD程序即可实现类 库文件的自动加载。

(if (not (= (substr (ver) 1 11) "Visual LISP")) (load "acad2012doc.lsp"))

(command "netload" "X: $\mid \backslash X X X\|X X\| X X \| C A D . d l l ")$ 
;; Silent load.

(princ)

\section{4. 实例效果}

本文采用VS2010+AutoCAD2012定制开发建设工程 配套绿地面积审核工具。通过配套绿地面积审核工具可快 速完成各类绿地实体面积的汇总统计及标注，如下图4所
示。其中(1)为定制开发的菜单, 该菜单功能按照3.2中功 能规划进行配置; (2)为按图层及实体位置批量标识各绿地 实体的面积，便于对绿地实体面积进行核查; (3)为不同折 算系数绿地实体面积分类统计结果; (4)为所有单个绿地实 体的编号及面积。这四项功能可以相互关联和配合, 可提 高绿地实体面积统计的效率和准确率。

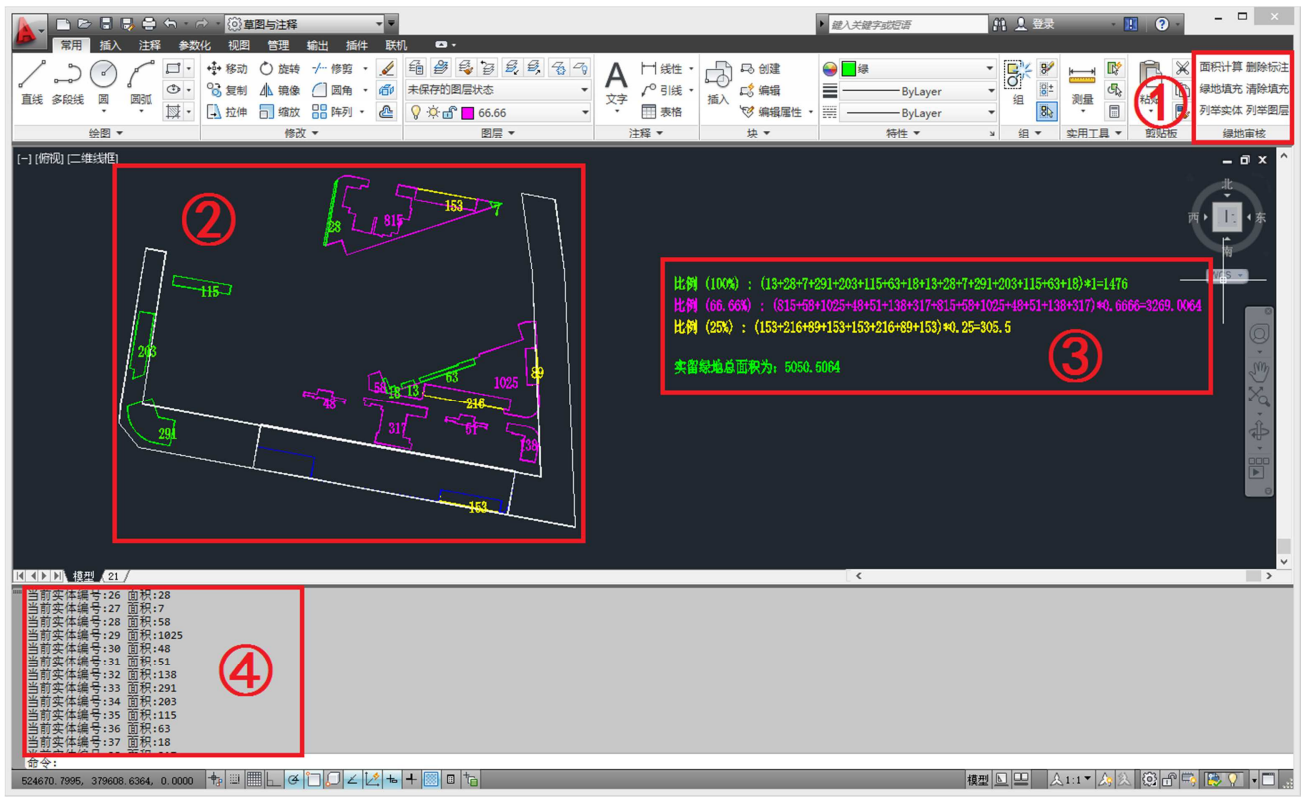

图4 配套绿地面积汇总统计工具界面。

\section{5. 结论}

随着经济社会和城市建设的快速发展，建设工程项目 配套绿地面积审核管理工作中也出现了一些新情况、新问 题。2015年武汉市制定了《武汉市建设工程项目配套绿地 面积审核管理办法》, 该办法对配套绿地面积审核管理工 作予以规范。本文以该办法为依据, 采用.NET API进行 AutoCAD二次开发, 快速完成了建设单位报送的规划设计 方案中各类绿地实体的面积分类汇总统计, 避免了传统人 工方式进行绿地实体面积统计易出错、难纠错的问题，大 幅地提高了配套绿地面积审核的工作效率。另外，在建设 工程项目规划条件核实阶段，还可使用该工具进行配套绿 地实施情况的实地测量成果的监督检查, 为建设工程项目 配套绿地批前和批后管理提供便利。

\section{参考文献}

[1] 李素英, 王计平, 任慧君. 城市绿地系统结构与功能研究综 述 $[J]$. 地理科学进展, 2010,29(3):377-379。

[2] 张纯. 生态园林城市绿地系统研究[D]. 杨凌: 西北农林科技 大学, 2011:1-3。
[3] 杜艳珊, 陈恒庆. 河南省平顶山市工程建设项目附属绿化工 程方案设计与审批[J]. 北京农业，2013(15):219-220。

[4] 宋威, 王正琼, 赵永亮. 使用C\#语言进行AutoCAD开发学 习初探 [J]. 测绘与空间地理信息，2010,33(4):225-227。

[5] 杨婷. CAD地形图高程信息快速提取的技术与实现[J]. 地 理空间信息，2015,13(1):162-164。

[6] 王小华, 程传录, 范宏涛. 基于AutoCAD .NET API的地形 图坐标转换实现方法研究 [J]. 测绘与空间地理信息, 2013,36(11)58-60。

[7] 李志超, 徐杉钰, 章波. 基于.NETAPI的CAD二次开发技术 在工程中的应用 [J]. 制造业自动化，2013,35(8):122-124。

[8] 侯颖, 何援军, 胡志刚. 非AutoCAD平台的DWG文件工程 信息提取技术 $[J]$. 计算机工程, 2003,29(11):180-183。

[9] 周波, 李俊峰, 刘帅. 自定义扩展AutoCAD属性信息的实现 [J]. 测绘与空间地理信息, 2014(37):65-67。

[10] 李霄楠, 于楷. 基于.NETAPI的AutoCAD二次开发实现快速 贯通误差预计 [J]. 北京测绘, 2014(02):67-71。 